\title{
Mapeamento de dados de um LMS para medida de construtos da distância transacional
}

\author{
Jorge L. C. Ramos ${ }^{1}$, João C. Sedraz Silva ${ }^{1}$, Alex Sandro Gomes ${ }^{2}$, \\ Fernando d. F. de Souza ${ }^{2}$, Rodrigo Lins Rodrigues ${ }^{3}$ \\ ${ }^{1}$ Universidade Federal do Vale do São Francisco \\ Juazeiro - BA - Brasil \\ ${ }^{2}$ Universidade Federal de Pernambuco \\ Recife-PE, Brasil \\ ${ }^{3}$ Universidade Federal Rural de Pernambuco \\ Recife-PE, Brasil. \\ \{jorge.cavalcanti, joao.sedraz\} @univasf.edu.br, \\ $\{$ asg, fdfd\}@cin.ufpe.br, rodrigo.linsrodrigues@ufrpe.br
}

\begin{abstract}
This paper presents a proposal for mapping the behavior data that represent the constructs of transactional distance (dialogue, structure and autonomy) in a Learning Management System (LMS). The aim was to describe and validate a set of variables under which these constructs can be measured, enabling the development of research in the area and obtaining these measures at any time during the course and without the need for questionnaires. The identification of an initial set was made by literature review, complemented with an analysis of the LMS database variables. Then a group of experts validated the initial set of attributes. The creation and validation of a final set of variables were made from the Confirmatory Factor Analysis (CFA), which pointed to each construct may be represented by a set of attributes obtained from LMS database.
\end{abstract}

Resumo. Este trabalho apresenta uma proposta para o mapeamento de dados de comportamento de usuários em um Learning Management System (LMS), em variáveis que representam os construtos da distância transacional (diálogo, estrutura e autonomia). O objetivo foi descrever e validar um conjunto de variáveis com as quais esses construtos podem ser medidos, permitindo o desenvolvimento de pesquisas na área, assim como a obtenção destas medidas a qualquer momento do curso e sem a necessidade de questionários. A identificação do conjunto inicial foi feita por revisão da literatura, complementada com uma análise das variáveis no banco de dados. Em seguida, um grupo de especialistas validou o conjunto inicial de atributos. A criação e validação de um conjunto final de variáveis foi feita a partir da Análise Fatorial Confirmatória (CFA), que apontou como cada construto pode ser representado por um conjunto de atributos obtidos a partir do banco de dados do LMS.

\section{Introdução}

O crescimento da Educação a Distância (EAD) exige análises orientadas por teorias para auxiliar o planejamento e a execução de cursos de maneira eficaz e eficiente. A EAD tem tido o êxito de ver o seu desenvolvimento histórico em paralelo com o desenvolvimento das suas teorias [Anderson e Dron 2012].

Com a evolução tecnológica, algumas das teorias da EAD precisam ser revisitadas e atualizadas, sobretudo em razão de novos recursos tecnológicos usados nos cursos, assim como a existência de novos métodos e processos que permitem um novo olhar sobre essas teorias, agregando uma importância ainda maior às mesmas.

Dentre essas várias teorias que norteiam a EAD, a Teoria da Distância Transacional [Moore 1973; 1993; 2013] é destacada por abrangência e atualidade, pois o seu autor tem 
V Congresso Brasileiro de Informática na Educação (CBIE 2016)

Anais do XXVII Simpósio Brasileiro de Informática na Educação (SBIE 2016)

buscado uma constante revisão da mesma, considerando a própria evolução da EAD, assim como outros pesquisadores que a consideram como uma teoria seminal e importante para a modalidade [Peters 2002]; [Chen 2001a]; [Zhang 2003]; [Sandoe 2005]; [Goel, Zhang e Templeton 2012]; [Huang et at. 2015].

Partindo dessa visão da necessidade de renovação de teorias, bem como aplicá-las no sentido de auxiliar no enfrentamento nos desafios da educação a distância, este trabalho enfoca a Teoria da Distância Transacional como ponto central do estudo, com o objetivo de propor uma nova abordagem para determinação dos seus construtos, a partir da identificação das diversas variáveis que formam esses construtos em um banco de dados do Learning Management System (LMS) Moodle. No capítulo 2 apresentamos a Teoria da Distância transacional. No capítulo 3 apresentamos o método adotado na pesquisa e no capítulo 4 são descritas as análises e a interpretação dos dados.

\section{Teoria da Distância Transacional}

Segundo Moore (1993), uma transação em educação a distância é a interação de professores e alunos em ambientes que têm a característica especial de serem espacialmente separados um do outro. Esta separação conduz a padrões especiais de comportamento de alunos e professores e afeta profundamente tanto o ensino, quanto a aprendizagem. Com essa separação, surge um espaço psicológico e comunicacional a ser transposto, um espaço de potenciais mal-entendidos entre as intervenções do professor e as do aluno. Este espaço psicológico e comunicacional é a distância transacional (DT).

A Teoria da Distância Transacional preconiza que os cursos a distância podem ser avaliados segundo uma medida dessa distância, a qual se baseia em três construtos: autonomia do aluno, diálogo e estrutura do curso [Moore 1993]. Estabelecer essa medida indicadora da distância transacional e assim como definir mecanismos ou procedimentos no sentido de reduzi-la, tem sido o foco de vários estudos desde a sua definição [Zhang 2003].

O diálogo é o construto usado para descrever a interação entre os participantes de um curso, com o objetivo de aperfeiçoar a aprendizagem. A estrutura expressa o nível de flexibilidade das estratégias pedagógicas e quanto o curso pode ser personalizado aos interesses dos estudantes. A autonomia avalia a liberdade que os estudantes possuem para decidirem sobre o seu ritmo e processo de aprendizagem [Moore 1993].

\subsection{Estratégias de medida da Distância Transacional e de seus construtos}

A Teoria da Distância Transacional tem sido motivadora de pesquisas que buscam desenvolver instrumentos para sua medição e, por consequência, atribuição de um valor ou conceito que serve como um possível indicador dessa distância.

Ser capaz de medir individualmente cada faceta da DT é fundamental para tornar mais efetivos os esforços de pesquisa na área, de modo a proporcionar aos atores da modalidade melhores condições de planejamento didático e monitoramento da aprendizagem [Sandoe 2005]. Para Goel, Zhang e Templeton (2012), a área problemática predominante na Teoria da Distância Transacional tem sido a sua medição geral e de seus construtos separadamente.

A definição da DT pode contribuir de forma relevante para o planejamento e execução de cursos online, pois seus construtos representam um indicativo do nível de comunicação e interação do aluno, o quanto a organização do curso é mais ou menos flexível para os alunos e de que forma eles estabelecem o seu próprio ritmo de aprendizagem [Huang et at. 2015].

Essa medida pode refletir uma condição ou um estado de um curso durante a sua realização, permitindo por exemplo, que professores e tutores percebam um distanciamento de determinados alunos e possam intervir no sentido de prever ou reverter situações de evasão de alunos do curso [Horzum 2011]. 
V Congresso Brasileiro de Informática na Educação (CBIE 2016)

Anais do XXVII Simpósio Brasileiro de Informática na Educação (SBIE 2016)

Estabelecer procedimentos e instrumentos de coleta de dados para buscar medir ou estabelecer indicadores da distância transacional tem sido um desafio relatado por diversos pesquisadores na literatura sobre a teoria. A maioria dos trabalhos tem abordagem quantitativa e adotam coletas de dados a partir de questionários com escalas tipo Likert [Chen 2001a; 2001b]; [Zhang 2003]; [Sandoe 2005]; [Goel, Zhang e Templeton 2012]; [Huang et at. 2015]. Também são feitas análises da transcrição de entrevistas abertas [Kanuka 2001]; e análises qualitativas [Kanuka, Collett e Caswell 2002]; [Ustati e Hassan 2013].

As medições baseadas em questionários ficam limitadas às opiniões dos respondentes e ao instante do curso no qual foram aplicados, o que pode, em algum momento, apresentar vieses que provoquem uma medição inadequada ou mesmo a faça de maneira incompleta.

Esses estudos apontam algumas limitações nesse método, de modo a não ser possível a sua generalização para diversas circunstâncias na qual ocorre a DT em outros cursos ou ambientes virtuais de aprendizagem distintos dos que foram analisados. Uma das limitações apontadas é a forma de coleta de dados sobre os quais é calculada ou medida a DT. Alguns trabalhos têm apresentado instrumentos para medição, mas sem conseguir, até o momento, contemplar todos os conceitos e propostas da teoria [Huang et at. 2015].

\section{Percurso Metodológico}

Este trabalho é parte de uma pesquisa em curso, que visa apontar estratégias alternativas e eficientes para medição dos construtos da DT e sua consequente utilização no planejamento e execução de cursos na EAD. O design deste estudo é apresentado na Figura 1.

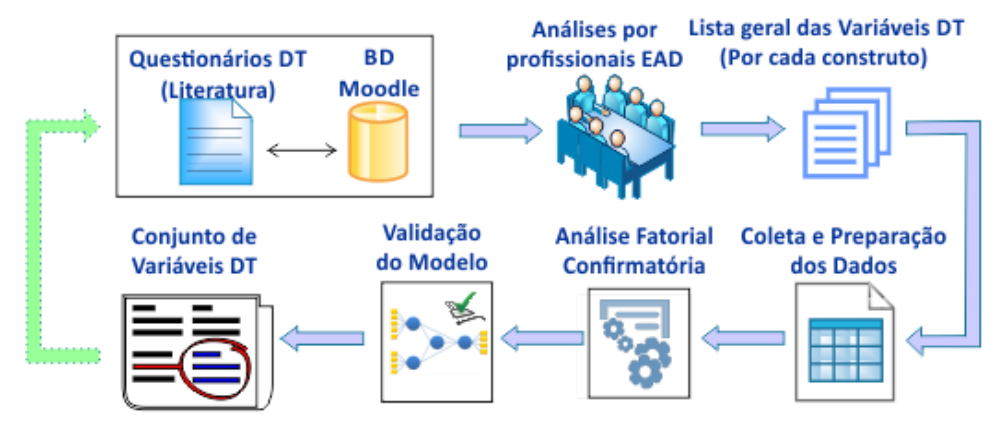

Figura 1: Etapas desenvolvidas neste trabalho (Fonte: Os autores).

Neste trabalho, foi estabelecido um modelo teórico provisório da DT, onde cada construto (variável latente) foi inicialmente representado por um conjunto de variáveis observadas, obtidas a partir do cotejamento de questionários usados em estudos sobre a distância transacional, com os registros de um banco de dados de um LMS. Um conjunto final das variáveis representativas da DT foi obtido a partir de um processo de análise multivariada dos dados. Nas seções seguintes serão detalhadas as etapas desenvolvidas neste estudo.

\subsection{Associação entre itens de questionários e variáveis do banco de dados do LMS}

Para que fosse desenvolvida e apresentada uma proposta alternativa para estabelecimento dos construtos da distância transacional, este estudo tomou como base 9 trabalhos que apresentaram como forma predominante da medição da DT com o uso de questionários. Os trabalhos com os questionários considerados para este estudo são listados na Tabela 1.

Tabela 1. Obras relacionadas a instrumentos de medição da DT usadas neste trabalho.

\begin{tabular}{|l|l|}
\hline \multicolumn{1}{|c|}{ OBRA } & \multicolumn{1}{|c|}{ AUTOR(ES) } \\
\hline $\begin{array}{l}\text { Dimensions of transactional distance in the world wide web learning } \\
\text { environment: a factor analysis }\end{array}$ & Chen (2001b) \\
\hline $\begin{array}{l}\text { Transactional distance in Web-based college learning environments: Toward } \\
\text { measurement and theory construction. }\end{array}$ & Zhang (2003) \\
\hline
\end{tabular}


V Congresso Brasileiro de Informática na Educação (CBIE 2016)

Anais do XXVII Simpósio Brasileiro de Informática na Educação (SBIE 2016)

\begin{tabular}{|l|l|}
\hline Measuring transactional distance of online courses: The structure component & Sandoe (2005) \\
\hline $\begin{array}{l}\text { Transactional Distance Theory: The Effect of Disseminating Educational } \\
\text { Messages to Frontline Nurses in an Acute Care Hospital Setting. }\end{array}$ & Hughes (2010) \\
\hline $\begin{array}{l}\text { Developing transactional distance scale and examining transactional distance } \\
\text { perception of blended learning students in terms of different variables. }\end{array}$ & Horzum (2011) \\
\hline Transactional distance revisited: Bridging face and empirical validity & Goel et al. (2012) \\
\hline $\begin{array}{l}\text { Relative Proximity Theory: Measuring the Gap Between Actual and Ideal } \\
\text { Online Course Delivery }\end{array}$ & Swart et al. (2014) \\
\hline $\begin{array}{l}\text { Measuring transactional distance in web-based learning environments: an initial } \\
\text { instrument development. }\end{array}$ & Huang et al. (2015) \\
\hline $\begin{array}{l}\text { Revisiting Zhang's scale of transactional distance: refinement and validation } \\
\text { using structural equation modeling. }\end{array}$ & Paul et al. (2015) \\
\hline
\end{tabular}

Diversas técnicas de análise dos dados foram usadas nesses questionários, tais como: análises fatoriais exploratória e confirmatória, modelagem de equações estruturais, análises das correlações e discriminantes, análise de variâncias, entres outras. No total, esses questionários geraram uma lista de 186 questões, categorizadas pelos respectivos autores em cada um dos 3 construtos da distância transacional.

O passo seguinte foi buscar estabelecer, para cada questão dessa lista, uma associação com os registros no banco de dados do LMS, estabelecendo um mapeamento preliminar de cada construto da DT. Associamos campos de tabelas do banco de dados do LMS a variáveis que poderiam servir para medir os construtos. Assim, foi identificado um conjunto inicial de variáveis representativas da DT, passíveis de serem extraídas diretamente do banco de dados, sem a necessidade de aplicação de questionários.

\subsection{Análise das variáveis por especialistas e relações com os construtos da DT}

Em escalas que medem características não-tangíveis, como crenças e atitudes, as opiniões de peritos em termos de sua relevância podem ajudar a estabelecer sua validade de conteúdo. A definição de como um conjunto de itens corresponde a determinados construtos de uma teoria pode ser feita com auxílio de especialistas usando, por exemplo, um questionário de avaliação e classificação dos itens [Hair et at. 2009].

Após a obtenção da lista inicial de variáveis, a mesma foi submetida por meio de questionário online, para professores atuantes na EAD em três instituições de ensino superior (IES) vinculadas à UAB. Além das questões pertinentes aos construtos, uma introdução com um breve resumo da Teoria da Distância Transacional foi apresentada para que os professores tivessem uma melhor contextualização das perguntas do questionário.

A intenção foi pedir que esses professores associassem cada uma das variáveis apresentadas a algum(ns) ou nenhum dos construtos da DT. Assim, para cada uma das variáveis listadas, o professor podia fazer associações da mesma com os construtos ou então, caso assim percebesse, assinalar a opção "nenhum” entre as respostas possíveis.

No questionário foi disponibilizado um campo tipo texto para o professor, caso assim desejasse, acrescentar algum comentário ou mesmo propor uma nova variável não listada no instrumento de coleta.

\subsection{Coleta e preparação dos dados de comportamento de usuários do LMS}

Foram analisados os dados referentes a 7 períodos de uma mesma turma de curso de pedagogia em uma IES pública, com 126 alunos matriculados, ofertado segundo diretrizes da Universidade Aberta do Brasil (UAB). Esse curso utiliza o LMS Moodle como ambiente virtual de aprendizagem.

A escolha pelo curso de pedagogia deu-se por ser o curso com maior número de alunos matriculados e também porque registrava o menor índice de evasões dentre os demais cursos ofertados pela IES. 
V Congresso Brasileiro de Informática na Educação (CBIE 2016)

Anais do XXVII Simpósio Brasileiro de Informática na Educação (SBIE 2016)

O passo seguinte foi a elaboração dos scripts em linguagem SQL, utilizando ferramentas gerenciais do SGBD PostGreSQL para a extração dos dados referentes às variáveis representativas dos construtos da DT no banco de dados do Moodle.

Após a extração, é feito o pré-processamento dos dados, como verificação e remoção de outliers, verificação de existência de missing values, para tornar os dados apropriados para o processo seguinte de Análise Fatorial.

\subsection{Análise Fatorial Confirmatória}

Com os dados ajustados e tratados, foi realizada a Análise Fatorial Confirmatória (CFA), como uma das técnicas de Modelagem de Equações Estruturais (SEM), cuja validação de construtos é umas das suas principais aplicações. Esse tipo de análise parte da premissa que já se tem uma teoria sobre quais variáveis medem quais construtos (fatores) e que se quer confirmar o grau de ajuste dos dados observados à teoria que foi baseada [Brown 2015].

A Analise Fatorial Confirmatória tornou-se uma análise multivariada popular entre pesquisadores em um intervalo de tempo relativamente pequeno, pois ela provê uma maneira conceitual atraente para se testar uma teoria. Se um pesquisador pode expressar uma teoria a partir de relações entre variáveis medidas (observadas) e construtos latentes (não observados), então a CFA pode avaliar quão bem a teoria ajusta-se à realidade, quando esta for representada por dados. A modelagem é feita a partir de um estimador definido conforme a normalidade ou não dos dados [Hair et at. 2009].

A partir da CFA, as relações entre as variáveis e construtos são modeladas em uma série de regressões lineares, utilizando para isso uma matriz de covariâncias. Assim, pode-se rever o modelo gerado e ajustá-lo seguidamente, ligando construtos e variáveis de outras formas na busca de melhores ajustes. Nessa abordagem, a carga fatorial de cada variável em relação ao seu construto representa o seu coeficiente na equação estrutural, como numa regressão linear.

Neste estudo, foram seguidas as recomendações de Jackson et at. (2009) para realização de uma CFA. As variáveis previamente definidas na etapa anterior com os especialistas (seção 3.2) foram utilizadas para validar os três construtos da teoria da DT.

É comum também mostrar um modelo fatorial confirmatório através de um path diagram em que quadrados representam variáveis observadas e círculos representam as variáveis latentes, facilitando uma rápida visualização e interpretação do modelo.

\subsection{Validação do Modelo}

Depois de o modelo CFA ser especificado, é realizada a avaliação do ajuste do modelo para verificar se o mesmo é consistente com os dados e se pode então representar a teoria em questão. Essa avaliação é feita a partir da medição dos principais índices de qualidade de ajuste do modelo; da análise das cargas fatoriais obtidas para cada variável; da identificação dos componentes nos quais não há ajuste ao modelo, através da análise dos resíduos e dos índices de modificação e da interpretação adequada dos parâmetros estimados [Hair et at. 2009].

Embora o modelo especificado possa promover um bom ajuste dos dados à teoria, a partir dos critérios de validação usados, ele não é o único. A CFA apenas confirma que ele é um entre diversos modelos possíveis e aceitáveis para os dados.

\subsection{Conjunto representativo de variáveis por construto da distância transacional}

Os resultados finais da CFA apontam para cada construto da DT, um conjunto representativo de variáveis que servirá como referência para novas medições e previsões, de acordo com o propósito educacional que se queira aplicar à teoria. Tal conjunto, embora reflita os dados analisados no estudo, deve permitir uma certa flexibilidade na adequação das variáveis para possibilitar seu uso em outros cursos EAD na mesma ou em outras IES. 
V Congresso Brasileiro de Informática na Educação (CBIE 2016)

Anais do XXVII Simpósio Brasileiro de Informática na Educação (SBIE 2016)

\section{Apresentação e interpretação dos resultados}

Para as análises deste estudo foi utilizado o software estatístico $\mathrm{R}^{1}$ com os pacotes lavaan $^{2} \mathrm{e}$ semPlot ${ }^{3}$ para o processo de análise fatorial confirmatória.

\subsection{Definição das variáveis e validação dos construtos por especialistas}

A partir da análise dos questionários listados na seção 3.1, um conjunto de 38 variáveis foi extraído e apresentado a professores que atuam cursos superiores na EAD em IES participantes do Sistema UAB. Foram dadas 40 respostas aos questionários. Apenas um professor apresentou sugestão para inclusão de duas novas variáveis para os construtos diálogo e autonomia, porém não foram incluídas por terem sido consideradas atendidas por outras variáveis já existentes. Algumas questões não puderam ser mapeadas, pois eram do tipo subjetivas ou de opinião, onde não foi possível a sua associação com os registros no LMS.

Por limitações de espaço neste trabalho, a lista inicial das variáveis e a respectiva atribuição de cada construto pelos professores foi disponibilizada como anexo digital no link: http://bit.ly/variaveisDTMoodle.

\subsection{Coleta e preparação dos dados de comportamento de usuários em LMS}

Foram gerados scripts em SQL que extraíram os dados das variáveis direto da base disponibilizada. A coleta de dados retornou um total de 5504 observações para cada uma das variáveis pré-selecionadas.

Os resultados foram verificados quanto à sua consistência e adequação à cada uma das variáveis coletadas. Para algumas variáveis, todos os valores retornaram zerados, indicando que não existia nenhum registro daquelas variáveis no banco de dados, em razão da estruturação do curso no ambiente virtual. Outras variáveis apresentaram um valor constante em todos os casos, sendo também descartadas das análises posteriores, por não influenciarem no modelo a ser estimado. Essas variáveis (Tabela 2) foram então eliminadas para as demais fases da pesquisa.

Tabela 2. Variáveis descartadas após verificação de consistência dos dados.

\begin{tabular}{|l|c|}
\hline \multicolumn{1}{|c|}{ VARIÁVEIS } & CONSTRUTOS \\
\hline Quantidade de sessões de chats sobre conteúdos disponibilizadas do curso. & $\mathrm{E}$ \\
\hline Quantidade de acessos do aluno à página de conteúdo da disciplina. & $\mathrm{A}$ \\
\hline Quantidade de acessos do aluno à página da agenda da disciplina. & $\mathrm{A}$ \\
\hline Quantidade postagens em chats feita pelo aluno no curso. & $\mathrm{E}$ \\
\hline Disponibilidade (existência) de página com a programa (conteúdo) da disciplina. & $\mathrm{E}$ \\
\hline
\end{tabular}

D - Diálogo, A - Autonomia, E- Estrutura - (*) Empate ou diferença menor ou igual a 10\% nas respostas.

Um problema detectado para extração de variáveis da componente "estrutura", foi o fato de muitos recursos do ambiente estarem configurados de maneira a não registrar o seu acesso pelos alunos, impossibilitando assim o seu registro no log do Moodle.

\subsection{Construção do modelo e validação dos construtos por CFA}

Para definição do modelo e validação dos respectivos construtos, inicialmente foi feito o teste de normalidade dos dados e, nesse caso, a suposição de normalidade multivariada não foi confirmada, a partir do resultado de 2 testes específicos (Mardia e Henze-Zirkler) conforme descritos no pacote $M V N^{4}$ do software R.

\footnotetext{
${ }^{1}$ https://www.r-project.org/

2 http://lavaan.ugent.be/

${ }^{3}$ https://cran.r-project.org/web/packages/semPlot/semPlot.pdf

${ }^{4}$ http://www.biosoft.hacettepe.edu.tr/MVN/
} 
V Congresso Brasileiro de Informática na Educação (CBIE 2016)

Anais do XXVII Simpósio Brasileiro de Informática na Educação (SBIE 2016)

Como a não normalidade dos dados foi verificada, foi preciso definir um estimador do modelo de CFA mais adequado, já que o método de Máxima Verossimilhança (ML Maximum Likelihood), mais usual neste tipo de análises, só pode ser aplicado em situações de normalidade dos dados. Neste caso, optou-se por usar o estimador dos Mínimos Quadrados Ponderados Diagonalmente (DWLS - Diagonally Weighted Least Squares).

Os métodos baseados na soma dos quadrados mínimos não requerem a normalidade e são também indicados para análise de variáveis categorizadas, mas exigem tamanho amostral maior ( $>400)$ [Hair et at. 2009], o que se encaixa nos dados coletados $(\mathrm{n}=5504)$.

Os primeiros testes para definição do modelo foram feitos com as 33 variáveis restantes, após a exclusão das variáveis listadas na Tabela 2, seguindo a distribuição por construto definida pelos professores descrita na Seção 4.1 deste trabalho. Os resultados deste modelo (Figura 2) já foram satisfatórios, considerando alguns dos índices de qualidade de ajuste comumente relatados na literatura.

dialogo = VAR01+VAR02+VAR03+VAR04+VAR05+VAR16+VAR19+VAR20+VAR21+VAR22+VAR25+VAR34+VAR39 estrutura $=\sim$ VAR08+VAR09+VAR14+VAR15+VAR23+VAR28+VAR30+VAR36

autonomia $=\sim$ VAR11+VAR12+VAR13a+VAR13b+VAR13C+VAR13d+VAR17+VAR18+VAR24+VAR26+VAR27+VAR31

Figura 2: Modelo inicial considerando as 33 variáveis restantes (Fonte: Os autores).

Entretanto, ao serem analisadas as cargas fatoriais das variáveis para verificar a sua importância em relação aos seus respectivos construtos, observou-se que algumas cargas apresentavam valor abaixo do mínimo recomendável (0.3) [Shevlin \& Miles 1998].

Além disso, os índices de modificação, que são indicadores obtidos para se verificar outras possíveis relações entre variáveis e construtos não especificados no modelo inicial, apontaram que a var26 teria uma influência muito mais positiva no modelo, caso fosse realocada do construto 'Autonomia' para o construto 'Estrutura'. Essa mudança pôde ser feita sem prejuízo com o que foi definido pelos professores, já que essa variável foi apontada para ambos os construtos pelos professores.

Dessa forma, optou-se por retirar as variáveis de baixa carga fatorial $(<0.3)$ e mudar a variável var26 para a 'Estrutura' e testá-lo novamente sob estas novas condições. Foram realizados quatro ciclos de CFA para se obter um modelo final satisfatório, apresentado na Figura 3.

dialogo = VAR01+VAR04+VAR05+VAR16+VAR19+VAR20+VAR21

estrutura $=\sim$ VAR08+VAR09+VAR23+VAR26+VAR28+VAR30

autonomia $=\sim$ VAR11+VAR12+VAR13a+VAR13b+VAR13c+VAR14+VAR17+VAR18+VAR24+VAR31

\section{Figura 3: Novo modelo após remoção e relocação de variáveis (Fonte: Os autores).}

Observamos que o modelo final foi reduzido para 23 variáveis, onde o construto 'Diálogo' teve uma redução de 13 para 7 variáveis significativas, em razão da similaridade ente algumas das suas variáveis. Já os construtos 'Estrutura' e 'Autonomia' perderam apenas 2 das variáveis orginalmente definidas. Os principais resultados desse modelo são apresentados na Tabela 3. Esses resultados apontaram a convergência do processo de estimação após 145 interações, o que indica para existência de pelo menos um modelo que se ajusta aos dados.

Para as variáveis latentes (construtos), são apresentados os valores estimados dos parâmetros de cada variável e respectivas cargas fatoriais de cada variável observada, além dos erros padrões associados.

$\mathrm{Na}$ avaliação do modelo, as seguintes medidas de qualidade de ajuste entre o modelo proposto e os dados da amostra foram utilizadas: indice $\chi 2 / g l$ (razão entre o qui-quadrado e $\mathrm{n}^{\circ}$ de graus de liberdade dos dados), CFI (Índice de Ajuste Comparativo), GFI (Índice de Qualidade do Ajuste), NFI (Índice de Ajuste Normado), TLI (Índice de Tucker-Lewis) e o RMSEA (Raiz do erro quadrático médio de aproximação). 
V Congresso Brasileiro de Informática na Educação (CBIE 2016)

Anais do XXVII Simpósio Brasileiro de Informática na Educação (SBIE 2016)

Tabela 3. Resumo dos resultados da CFA no modelo proposto.

\begin{tabular}{|c|c|c|c|c|c|c|c|c|c|}
\hline & Variáveis & Estimativa & Erro & Carga & & Variáveis & Estimativa & Erro & Carga \\
\hline \multirow{7}{*}{ 苗 } & VAR01 & 1.000 & & 0.604 & \multirow{10}{*}{$\begin{array}{l}\Xi \\
\vdots \\
z \\
z \\
0 \\
\vdots \\
\vdots\end{array}$} & VAR11 & 1.000 & & 0.513 \\
\hline & VAR04 & 6.099 & 0.154 & 0.459 & & VAR12 & 8.470 & 0.220 & 0.924 \\
\hline & VAR05 & 14.838 & 0.227 & 0.832 & & VAR13a & 53.157 & 1.402 & 0.789 \\
\hline & VAR16 & 0.936 & 0.019 & 0.405 & & VAR13b & 83.714 & 2.184 & 0.846 \\
\hline & VAR19 & 3.717 & 0.097 & 0.449 & & VAR13c & 75.495 & 2.014 & 0.796 \\
\hline & VAR20 & 12.875 & 0.186 & 0.827 & & VAR14 & 1.460 & 0.038 & 0.553 \\
\hline & VAR21 & 3.850 & 0.074 & 0.379 & & VAR17 & 0.476 & 0.013 & 0.608 \\
\hline \multirow{6}{*}{ 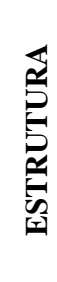 } & VAR08 & 1.000 & & 0.753 & & VAR18 & 220.319 & 5.711 & 0.924 \\
\hline & VAR09 & 0.370 & 0.006 & 0.654 & & VAR24 & 29.047 & 0.844 & 0.414 \\
\hline & VAR23 & 0.102 & 0.002 & 0.575 & & VAR31 & 51.913 & 1.429 & 0.740 \\
\hline & VAR26 & 0.546 & 0.011 & 0.555 & \multirow{3}{*}{\multicolumn{3}{|c|}{$\begin{array}{l}\text { Number of observations } \\
\text { Estimator } \\
\text { Degrees of freedom } \\
\text { P-value (Chi-square) }\end{array}$}} & \multirow{3}{*}{\multicolumn{2}{|c|}{$\begin{array}{l}5504 \\
D W L S \\
227 \\
0.000\end{array}$}} \\
\hline & VAR28 & 0.104 & 0.002 & 0.384 & & & & & \\
\hline & VAR30 & 0.143 & 0.002 & 0.694 & & & & & \\
\hline
\end{tabular}

A Tabela 4 apresenta os índices de ajuste do modelo obtidos, comparando-os com os valores recomendados na literatura.

Tabela 4. Indicadores de ajuste e qualidade do modelo.

\begin{tabular}{|c|c|c|c|}
\hline Indicador & $\begin{array}{c}\text { Valores recomendados } \\
{[\text { Hu \& Bentler 1999] }}\end{array}$ & $\begin{array}{c}\text { Valores recomendados } \\
\text { [Hair } \text { et at., 2009] }\end{array}$ & Valor obtido \\
\hline$\chi 2 / g 1$ & $<=2$ & $<=3$ & 29.05 \\
\hline CFI & $>0.95$ & $>0.90$ & 0.922 \\
\hline GFI & $>0.90$ & $>0.90$ & 0.940 \\
\hline TLI & $>0.95$ & $>0.90$ & 0.913 \\
\hline NFI & $>0.95$ & $>0.90$ & 0.920 \\
\hline RMSEA & $<=0.06$ & $<=0.07$ & 0.070 \\
\hline
\end{tabular}

O fato do primeiro indicador ter apresentado um valor acima do recomendado não inviabiliza a análise nem torna o modelo incompatível com os dados. Como esse índice é uma função matemática, que depende do tamanho da amostra e da diferença entre as matrizes estimadas e observadas do modelo, é comum para amostras grandes ( $\mathrm{n}>=500$ ) que esse valor seja alto, indicando uma possível rejeição do modelo, mesmo se a diferença entre as matrizes for mínima [Hu \& Bentler 1999] [Hooper 2008] [Hair et at., 2009]. Por isso outros indicadores de ajustes foram desenvolvidos e são amplamente utilizados em CFA, como os utilizados neste trabalho. Além desses apresentados, outros índices de ajustes também apresentaram valores relevantes neste estudo, confirmando a adequação dos dados analisados à teoria.

Na Tabela 5 são listadas as variáveis consideradas para cada construto, baseado no modelo ajustado aos dados analisados exibido na Tabela 3.

Tabela 5. Conjunto final de variáveis por construto, após ajustes no modelo.

\begin{tabular}{|c|c|c|}
\hline Var & Descrição & Construto \\
\hline VAR1 & Quantidade geral de postagens de um aluno em fóruns. & \multirow{7}{*}{ Diálogo } \\
\hline VAR4 & Quantidade geral de mensagens enviadas pelo aluno dentro do ambiente. & \\
\hline VAR5 & Quantidade geral de mensagens recebidas pelo aluno dentro do ambiente. & \\
\hline VAR16 & Quantidade de colegas diferentes para quem o aluno enviou mensagens & \\
\hline VAR19 & Quantidade de mensagens enviadas por aluno para professores pelo ambiente. & \\
\hline VAR20 & Quantidade de mensagens de professores recebidas por aluno dentro do ambiente. & \\
\hline VAR21 & Quantidade de mensagens de colegas recebidas por aluno dentro do ambiente. & \\
\hline VAR8 & Quantidade geral de recursos disponibilizados pelos professores por disciplina. & Estrutura \\
\hline
\end{tabular}


V Congresso Brasileiro de Informática na Educação (CBIE 2016)

Anais do XXVII Simpósio Brasileiro de Informática na Educação (SBIE 2016)

\begin{tabular}{|c|c|c|}
\hline VAR9 & Quantidade geral de atividades disponibilizadas pelos professores por disciplina. & \\
\hline VAR23 & Quantidade de atividades com prazos de resposta ou envio definidos pelo professor. & \\
\hline VAR26 & Quantidade de acessos de um aluno aos diferentes recursos (página web, vídeo, pdfs...). & \\
\hline VAR28 & Quantidade de fóruns de discussão disponibilizados sobre os conteúdos do curso. & \\
\hline VAR30 & Quantidade de sessões de web conferências disponibilizadas no curso. & \\
\hline VAR11 & Média semanal da quantidade de mensagens enviadas pelo aluno no ambiente. & \multirow{9}{*}{ Autonomia } \\
\hline VAR12 & Média semanal da quantidade de acessos do aluno ao ambiente. & \\
\hline VAR13a & Quantidade de acessos do aluno ao ambiente por turno (Manhã). & \\
\hline VAR13c & Quantidade de acessos do aluno ao ambiente por turno (Noite). & \\
\hline VAR13d & Quantidade de acessos do aluno ao ambiente por turno (Madrugada). & \\
\hline VAR14 & Quantidade de atividades entregues por um aluno no prazo, por disciplina. & \\
\hline VAR17 & Tempo médio diário de utilização da plataforma pelo aluno. & \\
\hline VAR26 & Quantidade de acessos de um aluno aos diferentes tipos de recursos disponibilizados. & \\
\hline VAR27 & Quantidade de acessos de um aluno aos diferentes tipos de atividades disponibilizadas. & \\
\hline
\end{tabular}

A relação final das variáveis por construto indica 7 variáveis significativas para o diálogo, 6 para estrutura e 10 para autonomia. Além disso, os resultados apontaram que existe uma covariância de 0.90 entre os construtos diálogo e autonomia, indicando uma boa inter-relação entre ambos. A covariância de 0.60 entre a estrutura e o diálogo também é representativa da relação entre esses construtos.

As variáveis representativas de cada construto podem ser coletadas em diferentes instâncias do Moodle ou até mesmo de outros LMS, já que representam registros básicos que o ambiente deve ter em seu banco de dados.

\section{Conclusões e trabalhos futuros}

A Teoria da Distância Transacional, ao longo de mais de 40 anos, vem estimulando pesquisas para aplicá-la de maneira a resolver ou atenuar problemas prementes da modalidade EAD. É uma teoria seminal e importante no contexto da educação a distância.

Este estudo apresentou uma proposta alternativa para obtenção dos construtos da distância transacional a partir da extração de dados diretamente do banco de dados do LMS Moodle. Esse BD é composto por mais de 250 tabelas, cada uma com vários campos e uma complexidade nos relacionamentos entre essas tabelas, atributos, chaves, entre outros.

A identificação de quais variáveis que compõem cada construto da distância transacional (diálogo, estrutura e autonomia) no BD do Moodle possibilitará a sua extração e utilização em outras instâncias do LMS e permitirá a continuidade de novas pesquisas relacionadas à DT nesse e em outros ambientes virtuais, usando inclusive processos mais avançados como a Educational Data Mining (EDM).

Outras vantagens são que os pesquisadores podem abrir mão de usar questionários para obter os indicadores da DT, assim como podem coletar dados em diversos momentos do curso. Embora o conjunto de variáveis da DT neste trabalho seja resultante do processamento dos dados de um curso especifico que utilizou o Moodle, o processo como um todo poderá ser replicado e utilizado em outros cursos, instituições e mesmo para diferentes LMS, desde que seja possível a extração dos dados relacionados com as variáveis da DT do seu banco de dados.

O mesmo conjunto de variáveis para cada construto foi utilizado em outros cursos e períodos distintos na IES e mostrou-se bem ajustado, com pequena variação no grupo de variáveis da estrutura.

Como continuidade deste estudo, serão testados modelos com outros cursos, buscando grupos de variáveis a partir dos perfis dos cursos e dos usuários. Há também uma intenção de tornar automática as coleta e análises das variáveis, a partir de plug-ins ou apps, com interfaces intuitivas para usuários, abstraindo toda a complexidade associada à coleta e análise dos dados. 
V Congresso Brasileiro de Informática na Educação (CBIE 2016)

Anais do XXVII Simpósio Brasileiro de Informática na Educação (SBIE 2016)

\section{Agradecimentos}

Esta pesquisa é apoiada pela FACEPE-APQ No. 0525-1.03/14. Os autores também agradecem ao apoio do NEAD/UPE e da PRPPGI/UNIVASF (Edital 18/2013). Alex Sandro Gomes é bolsista DT Nível 2/CNPq, processos n 310466/2012-1 e no 475634/2013-6.

\section{Referências}

ANDERSON, T.; DRON, J. Three generations of distance education pedagogy. The International Review of Research in Open and Distributed Learning, v. 12, n. 3, p. 80-97 2010.

BROWN, T. A. Confirmatory factor analysis for applied research. Guilford Publications 2015.

CHEN, Y.-J. Transactional distance in World Wide Web learning environments. Innovations in Education and Teaching International, v. 38, n. 4, p. 327-338, $2001 \mathrm{a}$.

CHEN, Y. J. Dimensions of transactional distance in the World Wide Web learning environment: a factor analysis. British Journal of Educational Technology, v. 32, n. 4, p. 459-470 2001 b.

GOEL, L.; ZHANG, P.; TEMPLETON, M. Transactional distance revisited: Bridging face and empirical validity. Computers in Human Behavior, v. 28, n. 42012.

HAIR, J. F. et at. Análise multivariada de dados. Bookman Editora 2009.

HORZUM, M. B. Developing Transactional Distance Scale and Examining Transactional Distance Perception of Blended Learning Students in Terms of Different Variables. Educational Sciences: Theory and Practice, v. 11, n. 3, p. 1582-1587 2011

HOOPER, D. et at. "Structural Equation Modelling: Guidelines for Determining Model Fit." The Electronic Journal of Business Research Methods Volume 6 Issue 1 2008, pp. 53-60.

HU, L.T.; BENTLER, P.M, "Cutoff Criteria for Fit Indexes in Covariance Structure Analysis: Conventional Criteria Versus New Alternatives," Structural Equation Modeling, 6 (1), 1-55. 1999

HUANG, X. et at. Understanding transactional distance in web-based learning environments: An empirical study. British Journal of Educational Technology 2015. ISSN 1467-8535.

HUGHES, W. G. Transactional Distance Theory: The Effect of Disseminating Educational Messages to Frontline Registered Nurses in an Acute Care Hospital Setting. 2010. Ph.D. Southeastern Louisiana University.

JACKSON, D. L., GILLASPY Jr, J. A., \& PURC-STEPHENSON, R.. Reporting practices in confirmatory factor analysis: an overview and some recommendations. Psychological methods, 14(1), 6. 2009

KANUKA, H. University student perceptions of the use of the Web in distance-related programs. The Canadian Journal of Higher Education, v. 31, n. 3, p. 49 2001. ISSN 0316-1218.

KANUKA, H.; COLLETT, D.; CASWELL, C. University instructor perceptions of the use of asynchronous textbased discussion in distance courses. The American Journal of Distance Education, v. 16, n. 3, p. 151-167 2002. ISSN 0892-3647.

MOORE, M. G. The theory of transactional distance. In: MOORE, M. G. (Ed.). Handbook of distance education. New York: Routledge 2013. cap. 5, p.66-85.

MOORE, M. G. Theory of transactional distance. In: (Ed.). Theoretical Principles of Distance Education. New York: Routledge, 1993. p.22-29.

MOORE, M. G. Toward a theory of independent learning and teaching. The Journal of Higher Education, p. 661 679, 1973. ISSN 0022-1546.

PAUL, R. C. et at. Revisiting Zhang's scale of transactional distance: refinement and validation using structural equation modeling. Distance Education, v. 36, n. 3, p. 364-382 2015.

PETERS, O. Distance education in transition: new trends and challenges. Bibliotheks- und Informationssytem der Universität Oldenburg 2002.

SANDOE, C. Measuring transactional distance of online courses: The structure component. PhD. University of South Florida. 2005.

SHEVLIN, M.; MILES, J. N. V. Effects of sample size, model specification and factor loadings on the GFI in confirmatory factor analysis. Personality and Individual Differences, v. 25, n. 1, p. 85-90, 1998.

SWART, W. et at. Relative proximity theory: Measuring the gap between actual and ideal online course delivery. American Journal of Distance Education, v. 28, n. 4, p. 222-240 2014.

USTATI, R.; HASSAN, S. S. S. Distance Learning Students' Need: Evaluating Interactions From Moore's Theory Of Transactional Distance. Turkish Online Journal of Distance Education, v. 14, n. 22013.

ZHANG, A. Transactional Distance in Web-based College Learning Environments: Toward Measurement and Theory Construction. PhD Thesis. VCU Retrospective. Richmond. 2003. 